\title{
Session VI: Estimating Likelihood and Exposure, Part II
}

\author{
Hector QUEMADA* \\ Dept. of Biological Sciences, Wood Hall, Western Michigan University, Kalamazoo Michigan 49004, USA
}

\section{PRESENTERS}

Pathway Analysis from the Perspective of the IPPC

Bill Roberts

Department of Agriculture, Fisheries, and Forestry

Canberra, Australia

Risk of Impact and Exposure of Monarch Butterflies to Bt Maize Pollen

Mark Sears

University of Guelph

Guelph, Canada

Environmental Fate: Degradation of Bioactive Proteins from GMO Maize

Joel Coats

Iowa State University

Ames, USA

Gene Flow Analysis into Wild/Weedy Relatives from Crops with Centers of Origin/Diversity in Tropical America

Zaida Lentini

CIAT (International Center for Tropical Agriculture)

Cali, Colombia

\section{INTRODUCTION}

Exposure or likelihood of occurrence of an identified hazard is a key factor used by regulators in determining the magnitude of risk. One example of exposure is the amount of gene flow between transgenic crops and their non-transgenic counterparts or their compatible wild relatives. Gene flow itself is not a hazard, but instead represents the exposure of the environment to transgenes and the potentially novel traits that they produce. In assessing the risk presented by transgenes, the amount of gene flow may vary geographically, and depends upon several factors, including climatic conditions, existing pollinators, and day length. The variability in levels of gene flow makes it sensible to make a worst-case assumption when estimating exposure from gene flow; that is, to assume that gene flow and introgression will occur to other compatible populations, particularly wild relatives. Consequently, the most important fact to establish is whether or not gene flow can occur. Determining the precise amount of gene flow and its geographic variation

*Corresponding author: hector.quemada@wmich.edu is less useful in risk assessment, particularly when the risks being assessed are potential long-term or evolutionary consequences, such as weediness or invasiveness, or impact on biodiversity.

However, refined estimations of exposure can be useful for other assessments of risk. These exposure estimates can include the likelihood of occurrence of critical steps along a pathway that might lead to a hazard, such as in the likelihood of establishment of a plant pest species. Another example is the estimate of exposure of non-target organisms to toxins produced by transgenic plants that allow them to be resistant to pests.

\section{PATHWAY ANALYSIS FROM THE PERSPECTIVE OF THE INTERNATIONAL PLANT PROTECTION CONVENTION}

With respect to genetically engineered organisms, the interest of the International Plant Protection Convention is focused on those genetically engineered organisms that might be injurious to plants or plant products. Since the Convention focuses on the organism's phenotype, not 
genotype, genetically engineered organisms are a subset of the organisms that are considered to have a potential for harm when introduced into a new environment. Roberts described a structured pest risk assessment approach similar to that presented in previous sessions in the symposium, which includes estimates of the likelihood of specific events occurring along a pathway leading to the establishment of a pest species. This pathway analysis may be used in both qualitative and quantitative assessments of risk.

As applied to the assessment of the risk of pest establishment, risk is defined as the consequences of a pest event (hazard), and the probability of a pest event occurring (exposure). The analysis of this probability emphasizes the pathway of entry. More specifically, the risk assessment phase of analyzing risk involves the following elements: pest categorization, probability of entry, probability of establishment, probability of spread, assessment of consequences, and finally conclusions about the level of risk and the regulatory actions that might be necessary.

Note that the majority of the elements in this approach have to do with probabilities of certain key events: entry, establishment, and then spread. However, assessment of consequences is not ignored, and presumably, even if probabilities are low for each step, if the consequences are severe, then an assessment might lead to the conclusion that the pest risk of an organism is too high to allow its introduction.

The subsequent two presentations described work to estimate the exposure of the environment to compounds produced by transgenes. Of particular interest from a biosafety perspective are compounds that have biochemical activity, such as proteins that protect plants against insect pests, or proteins that may have pharmaceutical applications that may remain intact and active in the environment.

\section{RISK OF IMPACT AND EXPOSURE OF MONARCH BUTTERFLIES TO Bt MAIZE POLLEN}

Sears described the approach taken to arrive at a quantitative estimate of exposure in the case of the non-target, monarch butterfly, combined with quantitative estimates of toxicity (hazard) derived from laboratory studies. First, the toxicological effects of expression of Cry $1 \mathrm{Ab}$ protein in $B t$ maize were determined. Expression of transgenic protein in various plant structures and the viability of protein in the environment were studied, along with the nature of the non-target insect (similarity with the pest species with respect to sensitivity, its likelihood of exposure, and the consequences to this insect's populations if affected). Furthermore, laboratory and field bioassays were used to investigate the effect of Cry1Ab protein, ingested via pollen, on growth and survival of monarchs. Laboratory bioassays covered a range of potential doses, from no pollen to extremely high concentrations, while field bioassays covered the range of more realistic pollen exposures. The field studies showed that under certain scenarios, a measurable $(23.7 \%)$ reduction in survival to adulthood was observed with first instar larvae continually exposed to $B t$ pollen.

Whether or not this posed a significant risk to monarch populations depended upon the potential for contact with $B t$ pollen by vulnerable populations and life stages. This determination depended upon information on whether crop and non-target range overlap significantly. This information in turn depended upon the gathering of sufficient knowledge of crop and non-target ecology, and accurate crop production statistics. Crop statistics provided information on corn acreage/arable land, the proportion Bt maize grown, which allowed calculation of the probability (proportion exposed) of monarch larvae exposed to $50 \%$ of the pollen shed period. Based on this information, $2.4 \%$ of the monarch population would be exposed to a dose of pollen that could have an impact on survival. A simple arithmetic calculation concluded that $0.6 \%$ of the monarch population was at risk from ingesting pollen expressing $B t$ protein.

\section{ENVIRONMENTAL FATE: DETECTION, QUANTIFICATION AND DISSIPATION OF GENE PRODUCT}

The presentation by Coats approaches the issue of exposure from the perspective of time - the rate at which proteins produced by transgenic plants are removed from the environment. Information was provided on ongoing work to determine the fate in soil and water of three proteins that are or could be expressed in maize for commercial purposes: Cry1F, and the pharmaceutical proteins, Escherichia coli enterotoxin subunit B (LTB), and Vibrio cholerae toxin subunit B (CTB).

With respect to Cry1F, the studies were initiated because of the current lack of quantitative data regarding environmental fate and flow of Bt proteins in the environment, and moreover, because of the paucity of data on the fate of Bt proteins in aquatic systems. Data were obtained from enclosed microcosms in the laboratory. The amount of Cry $1 \mathrm{~F}$ was measured by ELISA assay. The kinetics of dissipation in water and sediment was measured. The authors concluded that Cry $1 \mathrm{~F}$ dissipated rapidly in water and sediment, that some binding to sediment particles might occur, and that sediment could be a sink for Bt proteins.

The pharmaceutical proteins are of interest because of their potential to move out of a production system 
and exert biological effects outside of the field. (However, it was pointed out in the following discussion session that these proteins are likely to be present naturally). Extensive effort was spent to develop the methodology of extraction and measurement. Data obtained by measurement with ELISA still require verification by other methods such as HPLC.

\section{GENE FLOW ANALYSIS INTO WILD/WEEDY RELATIVES FROM CROPS WITH CENTER OF ORIGIN/DIVERSITY IN TROPICAL AMERICA}

The final presentation by Lentini brought the study of exposure back to the issue of gene flow. Lentini et al. studied the frequency of gene flow in two different crops, illustrating the different patterns of exposure that result from the biology and agronomic systems connected with each crop. The two crops studied were beans (Phaseolus vulgaris), a crop that would have transgenic events deployed in its center of origin and diversity; and rice (Oryza sativa), an introduced species with wild relatives in Latin America. Lentini also presented work modeled after the SIGMEA project in Europe, using maize as a system for modeling gene flow.

The study of gene flow in beans was presented as a case study using samples from the field and germplasm banks to reconstruct historic gene flow events. Wild-weed crop complexes of beans occur throughout much of Central and South America, providing opportunities for gene flow between crop and wild populations over time. There is much evidence of hybridization between crop and wild populations, and the dynamics of these hybrid populations has been studied. Molecular markers (microsatellites and chloroplast DNA polymorphisms), as well as trait markers, were used to assess the extent of gene flow between crop and wild populations, and the direction of that gene flow. The results indicate that the predominant direction of gene flow is from wild to cultivated populations. Agricultural practices and environmental conditions influence the survival of wild and hybrid populations.

The work in rice was initiated because of significant knowledge gaps concerning the wild-weedy crop complex in the Americas. Study of the relationship between $O$. sativa and $O$. glumaepatula, the only AA genome species of Oryza in the Americas, showed that the geographic distribution of $O$. glumaepatula overlapped with that of O. sativa, as did flowering times. Thus, an opportunity for crossing between these two species with compatible genomes exists. Whether this potential results in significant gene flow still requires investigation, since it was observed that hybrids between a Costa Rican ecotype of $O$. glumaepatula and cultivated rice were sterile. Molecular markers will be used to assess the historical level of exchange between these two species, using samples collected from areas where hybridization is likely.

Weedy rice in tropical America presents another biological entity that is not yet well characterized. The genetic and evolutionary origins of weedy rice in this region are still being studied and not yet well defined. Molecular and morphological traits have been used as a means to clarify the relationships between weedy rice, cultivated rice, and the other wild rice species in tropical America. Molecular markers have also been used to determine the frequency of gene flow between weedy and cultivated rice. These studies show that the direction of gene flow is predominantly from cultivated into weedy/wild, at a frequency of $10^{-4}$ to $10^{-3}$.

\section{IMPLICATIONS FOR THE FUTURE}

Up to the present, the genetically engineered crops that have been commercialized have gained regulatory approval often because their developers have been able to demonstrate no hazard or minimal hazard, thus resulting in conclusions of low risk. Future genetically engineered crops may not be able to demonstrate lack of hazard. For example, certain (not all) plants that express pharmaceutical compounds may be judged to present significant hazards to human health. Thus considerations of exposure become important, as in the case of field trials of such crops, where measures to reduce exposure to an acceptable level are required to manage the risk.

Qualitative exposure estimates have been instrumental in eliminating many risk issues, as in the cases of soybean and maize in the United States, where the lack of compatible wild and weedy relatives preclude the need for studying any potential impacts on the environment and biodiversity through outcrossing to such populations. However, more countries will be facing situations where the likelihood of gene flow to wild relatives is significant - a situation now facing those who are dealing with the deployment of transgenic rice in Asia, for example. In such cases, the estimation of exposure or likelihood of occurrence of a particular event (such as the outcrossing of a drought tolerance gene into a compatible weedy relative) will play a more significant role in determining whether to release a particular crop, and the circumstances of that release. 